\title{
Le MASCHERE DEL POTERE, DA MACHIAVELli A PASOlini
}

\author{
Sonia TROVATO \\ Università degli Studi di Verona
}

\begin{abstract}
En): By analysing Niccolò Macchiavelli's and Ferrante Pallavicino's work, this article investigates the evolution of a simulation and dissimulation culture linked to the character of the Prince, paying particular attention to the signs inscribed over bodies by government activity. A further, short evaluation of Pasolini's later work, and especially of Salò o le 120 giornate di Sodoma, enables a reflection about the contemporary representations of political power and of its hiding strategies.
\end{abstract}

Keywords (En): power ; masks ; politics ; obscenity ; fraud

Parole-chiave (It): potere ; maschere ; politica ; oscenità ; frode

Si la foule gouvernée peut se croire l'égale du petit nombre qui governe, alors in n'y a plus de gouvernement.

Le pouvoir doit être hors de portée de la compréhension de la foule des gouvernées.

(Joseph De Maistre)

\section{La (dis)simulazione del Potere}

In una lunga e densa intervista rilasciata a Saverio Lodato nel 2008, il magistrato siciliano Roberto Scarpinato ha sostenuto che «ll vero potere è sempre "osceno". Opera cioè nel "fuori scena" ( $o b$ scenum). Sulla scena, nei luoghi istituzionali viene inscenata una rappresentazione per il pubblico.» (LODATO, SCARPINATO, 2008: 31) L'intervista è confluita in un volume significativamente intitolato Il ritorno del Principe, in omaggio al capolavoro che Machiavelli compose durante il lacerante esilio nella tenuta dell' Albergaccio e nel quale viene per la prima volta proclamata la liceità della simulazione e della dissimulazione in virtù della ragion di Stato. L'interpretazione di Scarpinato, che vede nella figura del Principe l'emblema della "normalità" italiana (intesa come normalizzazione di comportamenti criminali), si unisce a considerazioni analoghe condotte da scrittori e critici contemporanei. Tra queste, una delle più trancianti porta la firma di Tabucchi, che ha indicato il segretario fiorentino come «il paradigma, e quasi il codice genetico, di un certo spirito italiano che attraversa i secoli» (TABUCCHI, 1998: 46), attribuendo a tale "spirito" una colpevole compiacenza verso il cinismo, promosso a fondamentale qualità per chi esercita il potere, ma anche per chi lo subisce. Senza la formulazione laica della teoria politica, difficilmente Torquato Accetto avrebbe potuto concepire il proprio trattato Della dissimulazione onesta, un manifesto del nascondimento prudente durante l'oppressione della Controriforma e della dominazione spagnola, salvato dall'oblio da Benedetto 
Croce $^{1}$, il quale ne consacrò la definitiva fortuna e lo piegò alla causa culturale degli antifascisti. In quanto elogio dell' «arte di pazienzia, che insegna così di non ingannare come di non essere ingannato» (ACCETTO, 1997: 67), l'opera si pone apparentemente su un piano di aperta antitesi rispetto al pensiero machiavelliano, che alla dissimulazione unisce la celebrazione della frode, ma numerose spie lessicali rivelano la lettura attenta che Accetto ha compiuto del Principe ${ }^{2}$. Con la recisione netta dei legami tra teoria politica e morale, Machiavelli ha, infatti, rivoluzionato il modo d'intendere l'esercizio del potere, tanto che, pochi anni dopo, il filosofo fiammingo Giusto Lipsio si esprime nei seguenti termini: «Siano dalla vita humana bandite Simulazione e Dissimulazione. Della vita privata è vero, della publica non è così, né altrimenti può fare chi abbia in mano tutta la Repubblica» (LIPSIO, 1604: 145-46). La cultura della simulazione/dissimulazione trova terreno fertile nel Cinquecento a causa del fiorire delle corti, sistemi che, come ha scritto argutamente Amedeo Quondam, si costituiscono «sulla base di un'antropologica coazione al teatro: rispetto all'occhio di un altro che vede» (QUONDAM, 2009: XVIII), e che assumono come «strategia quella del far vedere, del mostrare, del sembrare, sempre correlati a un occultare, celare, simulare/dissimulare» (QUONDAM, 2009: Ivi). Nei ventisei brevi capitoli del capolavoro machiavelliano, il Principe è protagonista di una vera e propria rappresentazione teatrale, sapientemente allestita grazie al «temperamento di drammaturgo» (GNOLI, SASSO, 2013: 23) dello scrittore rinascimentale, il quale può così concedere ai lettori-spettatori un punto di vista inedito: lo spazio al di là del sipario, quel luogo oscuro mai svelato dalla trattatistica politica precedente, ancora legata a una visione utopistica della teoria del governare, visione che è oggetto di ripetute sferzate polemiche da parte dell'autore ${ }^{3}$. Di seguito, ci si propone di analizzare, tramite l'opera e il pensiero di Machiavelli e di Pallavicino, l'evoluzione della figura del Principe tra il XVI e il XVII secolo, con una particolare attenzione ai segni che la pratica politica scrive sui corpi. In anni più recenti, le rielaborazioni artistiche più significative delle tecniche di nascondimento del potere si ravvisano nell'opera di Pasolini, in particolare in Salò o le 120 giornate di Sodoma: una rapida disamina del film consentirà così di riflettere sul progressivo affievolirsi dei tratti eroici e avventurosi che connotano le prime rappresentazioni letterarie del Principe.

\footnotetext{
${ }^{1}$ Cfr. Croce Benedetto (1928), Torquato Accetto e il trattatello Della dissimulazione onesta in: Croce Benedetto (1931), Nuovi saggi sulla letteratura italiana del Seicento, Bari, Laterza, p. 84-92.

${ }^{2}$ Per un'analisi delle suggestioni machiavelliane presenti nell'opera di Accetto cfr. ARNAUDO Marco (2009), L'altra dissimulazione: Accetto, Pallavicino, Machiavelli, Italica 86, p. 488-499.

${ }^{3}$ Nel capitolo XV, Machiavelli osserva, con biasimo, che «molti si sono immaginati republiche e principati che non si sono mai visti né conosciuti in vero essere. Perché gli è tanto discosto da come si vive a come si doverrebbe vivere che colui che lascia quello che si fa per quello che si doverrebbe fare impara più presto la ruina che la perservazione sua: perché uno uomo che voglia fare in tutte le parte professione di buono, conviene che ruini in fra tanti che non sono buoni». (MACHIAVELLI, 2013: 109-110). Lo scrittore pensa presumibilmente alla Repubblica di Platone, ma anche all'azione politica di Savonarola, che nel capitolo VI è citato come esempio negativo di profeta disarmato.
} 


\section{Lo "spettaculo" del Principe di Machiavelli}

Nell'omonimo saggio, il Principe è ripetutamente e perentoriamente esortato a mentire ai propri sudditi. Nel capitolo XVIII, dove è scritto che un buon principe debba essere un «gran simulatore e dissimulatore» (MACHIAVELLI, 2013: 125), l'elenco delle caratteristiche preposte al governo si chiude con un inciso eloquente, ossia che «A uno principe adunque non è necessario avere in fatto tutte le soprascritte qualità, ma è bene necessario parere di averle; anzi ardirò di dire questo, che, avendole e osservandole sempre, sono dannose, e parendo di averle sono utili» (Ivi: 126). Il motivo è chiarito poco dopo: «ognuno vede quello che tu pari, pochi sentono quello che tu sè» (Ivi: 127) e «el vulgo ne va preso con quello che pare» (Ivi: 128). Un sovrano che intenda costruire un regno stabile deve, pertanto, «parere piatoso, fedele, umano, intero, religioso» (Ivi: 126) e «avere gran cura che non gli esca mai di bocca cosa che non sia piena delle soprascritte cinque qualità» (Ivi: 127). L'esibita professione di fede è indicata come la più necessaria, poiché la religione è un instrumentum regni imprescindibile in Machiavelli ed è probabilmente l'indice più manifesto dell'emancipazione che egli compie dalla tradizione medievale.

Già nelle prime battute del libro viene affermato il principio per il quale serve il «favore de' provinciali a entrare in una provincia» (Ivi: 11), rinforzato, nel capitolo XX, dall'idea che «la miglior fortezza che sia è non essere odiato dal populo» (Ivi: 156): è indispensabile che il Principe consacri la propria attività politica all'ottenimento del consenso popolare e che si risparmi «quelle cose che lo faccino odioso o contennendo» (Ivi: 129), che sono, in primo luogo, le caratteristiche dell'incostanza, della leggerezza, dell'effeminatezza, della pusillanimità, dell'irresolutezza. Per scongiurare il rischio di una sommossa, i provvedimenti particolarmente sgraditi vanno delegati ad altri organismi. Ancora, un principe che intenda essere stimato deve adoperarsi nelle «grande imprese e dare di sé rari essempli» (Ivi: 179), dissimulando paure e viltà e non sottraendosi mai di fronte a una contesa. È, inoltre, opportuno che esalti gli uomini virtuosi ed eccellenti in un'arte, anche se questa non gli suscita interesse, che istituisca feste e spettacoli per il popolo e che vi partecipi sporadicamente, reprimendo temporaneamente la propria condizione sociale privilegiata, senza però abdicare al decoro e alla dignità. Il Principe deve, in sostanza, «dissimulare l'animo suo» (Ivi: 47), come seppe fare Cesare Borgia, assurto, nel capitolo a lui dedicato, a esempio massimo di principe nuovo capace di raggiungere i propri scopi con l'inganno e con la violenza. Figlio del papa Alessandro VI, Cesare Borgia riuscì, appoggiato dal padre ma soprattutto grazie a un'innata spregiudicatezza, a spodestare i signori di Romagna e a divenire vicario di Imola e di Forlì, gonfaloniere della Chiesa e duca di Valentinois, istituendo ex novo uno stato personale, che però crollò a causa di una «estraordinaria e estrema malignità di fortuna» (Ivi: 43), poiché alla morte del pontefice sopraggiunse la sua malattia, che gli impedì di difendersi col dovuto vigore dai nemici. Machiavelli aveva già narrato le gesta di Borgia nella cronaca del Modo che tenne il Duca Valentino per ammazar Vitellozo, Oliverotto da Fermo, il signor Pagolo e il duca di Gravina Orsini in Senigaglia, testo nel quale il futuro signore della Romagna è descritto come un «grandissimo simulatore» 
(MACHIAVELLI, 1997: 18), in grado di silenziare i rivali con un truce assassinio che l'autore descrive in toni quasi ammirati. Nel «piccolo volume» (Machiavelli, 2013: 4) dato in dono ai Medici, Borgia è celebrato come il principe più capace dei tempi moderni, per la sua encomiabile versatilità, la sua propensione all'inganno e l'intenso timore che seppe incutere nei contemporanei. Nell' affrontare la disputa «s'egli è meglio essere amato che temuto o è converso» (Ivi: 117), lo scrittore fiorentino conclude che è meglio essere temuti che amati, perché l'amore è normalmente generato da un sentimento di riconoscenza che si esaurisce presto, essendo la natura umana cattiva, simulatrice e dissimulatrice ${ }^{4}$, mentre «il timore è tenuto da una paura di pena che non ti abbandona mai» (Ivi: 119). Ottenuti i territori per cui ha combattuto crudelmente, il Principe deve, con azioni esemplari, mostrarsi pietoso per redimersi agli occhi del suo nuovo popolo. La crudeltà è dunque tollerata e, anzi, auspicata se utilizzata a scopi di conquista, ma deprecata se diventa un sistema di governo e se, quindi, si protrae oltre la presa di potere. Cesare Borgia fu un maestro anche nell'affinare questa "apparenza di crudeltà". Dopo aver conquistato Imola e Forlì, il duca decise di emanciparsi dalle armi e dalla fortuna altrui: attraverso un'efficace campagna di corruzione, egli privò i Colonna e gli Orsini di molti sostenitori, che passarono dalla sua parte. Mentre i Colonna erano già stati arginati, gli Orsini tentarono di ordire un complotto ai suoi danni, riunendosi a Magione, nel territorio di Perugia, e scatenando così una serie di eventi avversi al duca, il quale ebbe la meglio grazie all' appoggio dei francesi. Seguì il barbaro assassinio dei nemici:

E ritonatoli la reputazione, né si fidando di Francia né di altre forze esterne, per non le avere a cimentare si volse alli inganni; e seppe tanto dissimulare l'animo suo che li Orsini medesimi mediante il signore Paulo si riconciliorno seco - con il quale il duca non mancò d'ogni ragione di offizio per assicurarlo, dandoli danari, veste e cavalli - tanto che la simplicità loro gli condusse a Sinigaglia nelle sua mani. (Ivi: 47)

Sedati i dissapori con la ferocia soprascritta, Borgia era pronto a governare la Romagna e il ducato di Urbino; nel seguente passaggio, Machiavelli illustra le modalità con cui egli pacificò il territorio romagnolo, liberandolo da governi lassisti e incapaci attraverso il governo autoritario di Remirro de Orco, che fu poi sacrificato sulla pubblica piazza:

Presa che ebbe il duca la Romagna - e trovandola suta comandata da signori impotenti, e' quali più presto avevano spogliati e' loro sudditi che correttoli, e dato loro materia di disunione, non d'unione, tanto che quella provincia era tutta piena di latrocini, di brighe e d'ogni altra ragione di insolenza - iudicò fussi necessario, a volerla ridurre pacifica e ubbidiente al braccio regio, dargli buono governo: e però vi prepose messer Rimirro de Orco, uomo crudele e espedito, al quale dette plenissima podestà. Costui in poco tempo la ridusse pacifica e unita, con grandissima reputazione. Di poi iudicò il duca non essere necessaria sì eccessiva autorità perché

\footnotetext{
${ }^{4}$ «Perché degli uomini si può dire questo, generalmente, ch'e' sieno ingrati, volubili, simulatori e dissimulatori, fuggitori de' periculi, cupidi del guadagno» (MACHIAVELLI, 2013: 118). Il concetto è ribadito nei Discorsi sopra la prima deca di Tito Livio, dove si può leggere che «è necessario a chi dispone una republica ed ordina leggi in quella presupporre tutti gli uomini rei, e che li abbiano sempre a usare la malignità dello animo loro, qualunque volta ne abbiano occasione» (MACHIAVELLI, 1997: 207), «sendo gli uomini più proni al male che al bene» (Ivi: 224).
} 
dubitava non divenissi odiosa, e preposevi uno iudizio civile nel mezzo della provincia, con uno presidente eccellentissimo, dove ogni città vi aveva lo avvocato suo. E perché conosceva la rigorosità passata avergli generato qualche odio, per purgare li animi di quelli populi e guadagnarseli in tutto, volse mostrare che, se crudeltà alcuna era seguita, non era nata da lui ma da la acerba natura del ministro. E presa sopra a questo occasione, lo fece a Cesena una mattina mettere in dua pezzi in su la piazza, con un pezzo di legne e uno coltello sanguinoso accanto: la ferocità del quale spettaculo fece quegli popoli in uno tempo rimanere satisfatti e stupidi. (Ivi: 48-50)

Il duca esibisce pubblicamente il cadavere martoriato del suo dispotico gregario, simulando una presa di distanza dalle sue azioni riprovevoli, che ha, in realtà, egli stesso ordinato. Machiavelli definisce questa sezione del racconto «degna di notizia e da essere da altri imitata» (Ivi: 47), compiacendosi di queste azioni fraudolente e affrancandosi, così, dal pensiero dantesco, che alla frode riservava la zona più bassa dell'Inferno. D'altro canto, la frode trova una seconda legittimazione in un altro passo del Principe, nel quale il suo autore, domandandosi «Quanto sia laudabile in uno principe il mantenere la fede e vivere con integrità e non con astuzia» (Ivi:123) e commentando i vantaggi che molti grandi principi (Cesare Borgia, Francesco Sforza o Ferdinando il Cattolico) hanno ottenuto tenendo in poco conto la "fede", arriva a convenire che le promesse vadano adempiute quando non intralciano gli interessi del sovrano, il quale, facendosi scudo con l'innata malvagità dell'uomo, non faticherà a trovare moventi validi alla propria infedeltà: «Pacta sunt servanda è un principio costitutivo della politica buona, non della buona politica» (GNOLI, SASSO, 2013: 28).

La riflessione machiavelliana sulla doppiezza del potere (che ritorna, con strategie retoriche analoghe, anche nei Discorsi e nell'Arte della guerra) è da un lato il riflesso di un tratto tipico della cultura e della letteratura rinascimentale, ossia quello della reversibilità di ogni valore; dall'altro, costituisce un effetto tangibile dell'instabilità politica dell'Italia assediata dalle invasioni straniere, che trovano una legittimazione formale con la pace di Cateau-Cambrésis (1559), la quale sancisce ufficialmente il declino culturale e politico della penisola. Gli strumenti istituzionali di cui si dota la Chiesa postridentina per arginare ogni forma di dissenso e per disciplinare rigidamente l'espressione artistica implicano un ulteriore inasprimento della connotazione negativa della quale è investita la sfera politica. La censura e il clima di sospetto compromettono progressivamente l'incisività degli intellettuali e dei pensatori politici, «costretti ad accettare un ruolo sempre più subalterno nei confronti dei detentori del potere» (BALDINI, BATTISTA, 1997: 394). Per tutte queste ragioni, «nelle ricostruzioni storiche del tardo Cinquecento e del secolo XVII, la politica di governo e di corte si configura spesso con tratti particolarmente marcati di intrigo, doppiezza, astuzia, simulazione, crudeltà, ambizione personale oppure come un ripiegamento nella rassegnazione e nella rinuncia, nell'adulazione e nel servilismo» (VILLARI, 1987: $3-4)$. 


\section{La commedia dell'arte di governo: Il Principe ermafrodito}

Una delle opere che più rivela le storture del sistema politico cinquesecentesco è Il Principe ermafrodito (1640), frutto dell'estroso genio del giovane Ferrante Pallavicino, parmense canonico regolare che, a causa dei suoi libelli anticlericali e dei suoi romanzi irriverenti, venne tratto con l'inganno nel territorio papale di Avignone e decapitato a soli ventott'anni. Il libro fa parte di un «gruppo di testi che, utilizzando il codice del romanzo eroico-elegante o eroicocavalleresco, propone riflessioni e osservazioni sulla natura della politica» (COLOMBI, 2005: 20). La trama, ispirata a un testo teatrale spagnolo, è piuttosto intricata: Arlindo, re di Sicilia, alla nascita di una figlia femmina e alla notizia di una precoce sterilità della moglie, per aggirare il divieto di successione femminile imposto dalla legge salica e scongiurare il rischio di dispersione del regno, «cangiò a voglia sua questa prole» (PALlavicino, 2005: 49), imponendo alla figlia un'identità maschile. La fanciulla fu «alimentata con credito tale, imbevuto in ella medesma, in guisa che stimò d'esser uomo» (Ibidem). Riconoscendo i propri tratti anatomici in un ritratto di Venere che le venne presentata come la più bella delle dee, chiese spiegazioni al padre, il quale, pur svelando l'inquietante intrigo, le spiegò di aver agito per il bene del regno e di aver voluto rimediare con l'inganno ai falli della Natura e della Fortuna: «Non altri che me stesso volli fabro delle proprie contentezze, vedendo che l'architetto della natura falliva non incontrando i miei disegni» (Ivi: 53) è l'espressione con cui il re tenta di scagionarsi e con cui Pallavicino indirizza una frecciata satirica all'antropocentrismo rinascimentale, poiché chi abita nell'Italia controriformistica può difficilmente reputarsi l'artefice del proprio destino. Dopo essere stata ammonita dal padre affinché non si mostrasse effeminata e si tenesse lontana dalla passione amorosa, la giovane donna iniziò ad amoreggiare fintamente con la Duchessa di Prato Bello, così da rinforzare il proprio travestimento maschile. Quando, però, vide l'effigie di Alonso, valoroso cavaliere di cui la Duchessa era perdutamente innamorata, ne rimase a sua volta folgorata. Col pretesto di volerlo incontrare per conoscere il proprio rivale in amore, la Principessa lo convocò a corte e lo trasformò nel suo più fedele servitore. Non dimenticando la «giudiziosa prudenza con cui l'interesse di stato la necessitava a non iscouprirsi donna» (Ivi: 96), escogitò un piano per mettere alla prova la reciprocità dei loro sentimenti: inventò, cioè, l'esistenza di una misteriosa sorella gemella rinchiusa nelle segrete del palazzo in seguito alle nefaste visioni di un oracolo, che aveva predetto che la principessa avrebbe messo a rischio il regno per l'innamoramento verso un principe straniero. Con una serie di particolari ben tratteggiati, il Principe «autenticava la frode» (Ivi: 82) ${ }^{5}$ e si apprestava a diventare l'Ermafrodito, dato che avrebbe dovuto indossare la doppia veste di uomo e di donna. L'intrigo si infittì ulteriormente con l'arrivo a corte di Corgille, principessa di Napoli e promessa sposa del Principe-Principessa, venuta

\footnotetext{
${ }^{5}$ Il carattere ossimorico dell'affermazione rileva come la visione pessimistica e paradossale della letteratura pallaviciniana implichi la caduta di ogni strumento conoscitivo di distinzione tra autentico e inautentico. Lo si può constatare anche nell'espressione «confessione mista di veritadi e di menzogne» (PALLAVICINO: 70), dove il termine "confessione" è del tutto privato del proprio tratto etimologico e del proprio significato originario.
} 
sotto spoglie maschili perché spinta dalla curiosità verso il futuro marito. Notando una particolare sintonia tra il Principe e Alonso, la giovane si insospettì e iniziò a pensare che il cavaliere fosse in realtà una donna. Messo alle strette dal Re, il quale era stato allertato da Corgille, Alonso decise di assecondare le loro congetture e di fingersi di sesso femminile, sperando, in tal modo, di avere più occasioni di incontrare la Principessa, non potendo sapere che l'Ermafrodito avrebbe invece dismesso i panni della finta sorella. Dal canto suo, Corgille decise di rivelarsi e fece pressioni sul re perché affrettasse le sue nozze col Principe e organizzasse un matrimonio con un valente cavaliere anche per Alonso (ormai creduto Clarice), temendola ancora come rivale in amore. L'innaturalezza dell'ambiente di corte sembrò trionfare: la Principessa, donna, si sarebbe sposata in qualità di uomo, e Alonso, uomo, l'avrebbe fatto in qualità di donna. Ma l'Ermafrodito e Alonso si confidarono l'uno con l'altro: il primo svelò l'arcano della sorella inesistente e il secondo raccontò di essere il principe di Castiglia fuggito dal suo regno in seguito a un complotto di corte. Con un ulteriore inganno, i due riuscirono ad avere la meglio sulla ragion di Stato: l'Ermafrodito fece leva sui sentimenti che la Duchessa aveva iniziato a nutrire nei suoi riguardi affinché questa intercedesse presso il Consiglio del regno, dove sedevano alcuni parenti, e facesse abrogare la legge salica. A quel punto, la Principessa e Alonso si dichiararono pubblicamente e, grazie a un efficacissimo discorso e all'abolizione della norma, poterono convolare a nozze senza perdere la corona.

Nel romanzo pallaviciniano, i sentimenti, con il finale, sembrano vincere le subdole storture dell'ambiente di corte, ma la soluzione elaborata dall'Ermafrodito è ascrivibile alla stessa logica che ha guidato le azioni del Re, ossia all'idea, molto machiavelliana, secondo cui «chi non sa mentire, non sa regnare» (Ivi: 52). La figlia di Arlindo avrebbe potuto, infatti, sposare Alonso rinunciando al potere, e invece «Machinava l'imaginazione modo di non perdere il Regno nel goder l'amante. L'interesse di regnare ha conformità di possanza co' desideri d'amore» (Ivi: 87). L'espediente narrativo serve a Pallavicino ad articolare la propria condanna «all'etica della dissimulazione e dell'ostentazione, in quanto dietro di essa si vede solo una volontà di potere e di sopraffazione che costringe l'uomo e i suoi rapporti all'innaturalità» (COLOMBI, 2005: 34). Servendosi di molti temi tipici della cultura barocca, tra i quali compare anche il doppio, il chierico anticlericale evidenzia la deriva morale cui è approdata la corte, in quanto, tentando di sottomettere persino l'anatomia alle logiche del potere, «oltre a stravolgere i rapporti umani, conduce l'uomo a confondersi col suo ruolo sociale» (Ivi: 30). In tal senso, l'Ermafrodito non è mai apostrofato col vero nome (che non è neppure dichiarato), poiché cambia denominazione a seconda del travestimento e si identifica totalmente con il ruolo che riveste nel sistema di governo. Tutti i personaggi, d'altra parte, sono scarsamente caratterizzati e somigliano alle maschere della Commedia dell' arte, con un ruolo fisso, prestabilito e ripetitivo che acquista significato solo in virtù della funzione ricoperta nell'ambiente autoreferenziale della corte, il teatro di una messinscena ormai istituzionalizzata:

La corte, in questo genere di romanzi, è sempre lo spazio in cui l'eroe deve superare prove per affermare le proprie virtù [...]. Nella corte di Pallavicino, invece, le prove che devono superare i suoi eroi richiedono sempre e comunque una sola dote, quella di saper fingere, e così facendo 
l'autore lascia intendere esplicitamente di non celebrare, ma di criticare i non-valori della realtà etico-sociale rappresentata.

Evidente risulta dunque la posizione pallaviciniana secondo cui l'esercizio della politica non è altro che un gran teatro, realizzato per conservare il potere e i privilegi ad esso connessi, e che nel far ciò esso falsifica la realtà e distrugge i valori umani, svuota cioè la vita di umanità e di rispetto (Ivi: 33).

Un altro aspetto da considerare è che, da un punto di vista stilistico e formale, Pallavicino assume il codice del romanzo eroico-cavalleresco: ci sono, dunque, un intrigo politico e uno sentimentale. Ma nel Principe ermafrodito, l'amore non mette in moto una peripezia amorosa, è presentato come un istinto nocivo che mina le fondamenta della ragion di Stato e che deve, quindi, essere incasellato a forza in una dimensione esclusivamente politica della vita ${ }^{6}$. Dal canto suo, la politica non presenta tratti epici o eroici, essendo ridotta a una scienza meccanicista che ha, come scopo esclusivo, la propria perpetuazione. All'eroe del romanzo cavalleresco è in genere richiesto il superamento di una prova per affermare la propria virtù, mentre nel romanzo in questione l'unica virtù richiesta è l'attitudine alla menzogna. Inoltre, l'eroe cavalleresco è in perenne movimento, mentre l'eroe pallaviciniano non si muove dalla corte, che è presentata come un luogo asfittico e innaturale, che recide ogni vincolo familiare, amicale e solidale ${ }^{7}$. Bisogna notare, infine, che, a differenza di quanto avviene nella mitologia classica (dove l'ermafrodito, il figlio di Ermes e di Afrodite, può sperimentare entrambi i generi sessuali), nell'opera di Pallavicino la doppia identità sessuale è un travestimento e non un dato anatomico.

L'irriverente giovane scrittore, nascondendosi dietro a trame e contenuti apparentemente leggeri e disimpegnati, lancia un incisivo j'accuse al Potere, così da tentare di mettere fine alla «cecità indotta dallo stesso potere al fine di perpetuarsi» (LODATO, SCARPINATO, 2008: 16). Machiavelli nel Cinquecento aveva per primo reso noti gli spietati e brutali meccanismi della politica, accettati solo perché coerenti con una visione laica e terrena dell'arte di governo e di una ragion di Stato sempre più incerta. Un secolo dopo, quando ormai il capolavoro del segretario fiorentino compare da anni nell' Indice dei Libri proibiti, Pallavicino diventa l'eretico portavoce di un disincanto inquieto nei confronti della pratica governativa: «la fase eroica della "costruzione degli Stati" (quella simboleggiata da Machiavelli e dal "suo" duca Valentino) è da tempo terminata ed è stata sostituita da quella ben più piatta, ma parimenti necessaria, della "conservazione" di ciò che è stato conquistato» (BALDINI, BATTISTA, 1997: 395). Così, l' ambiguità del potere viene trasformata in un fattore di disordine sessuale, che coinvolge e sconvolge l'identità di genere e che incide sul corpo delle piaghe insanabili.

\footnotetext{
${ }^{6}$ Non a caso, nell'opera viene usata l'espressione «teatro degli affetti» (Ivi: 85).

${ }^{7}$ Cfr. Ortolani Donata (1978), Potere e violenza nel romanzo del Seicento, Catania, Pellicano.
} 


\section{L'eredità della teoria politica cinque-secentesca nel pensiero contemporaneo: Salò o le 120 giornate di Sodoma e lo stupro dei corpi}

La problematica del corpo violato dal potere diventa una tematica costante nell'ultimo Pasolini e in particolare nella sconvolgente riscrittura dell'opera sadiana, ossia Salò o le 120 giornate di Sodoma (1975), dove il Principe si frammenta in quattro figure diverse affiliate alla Repubblica Sociale Italiana: il Duca (potere nobiliare), il Monsignore (potere ecclesiastico), l'Eccellenza (potere giudiziario), il Presidente (potere economico). I quattro dispotici libertini decidono di dare vita a un progetto mostruoso, trasgressione segreta di tutti i proclami bigotti dell'ideologia nazifascista: si trasferiscono in una suntuosa villa, sposano le reciproche figlie, che poi diverranno schiave, commissionano una caccia di giovinette e giovinetti strappati dagli ambienti antifascisti e dissidenti, e instaurano una vera e propria dittatura sessuale, che sottopone le vittime a ogni tipo di degradazione, comprese la coprofagia e la necrofilia. Quei «corpi [...] ci appartengono», afferma il Duca, proclamando subito dopo un principio ribadito da Pasolini, e cioè che «La sola, vera anarchia è quella del potere», dato che è il potere stesso a stabilire indebitamente il discrimine tra lecito e illecito e a derogarlo a seconda delle proprie logiche utilitaristiche. Lo scrittore pensa naturalmente a un altro potere, ossia al potere consumistico, che considera ben più pervasivo e omologante della dittatura fascista. «Nessun centralismo fascista è riuscito a fare ciò che ha fatto il centralismo della civiltà dei consumi» (PASOLINI, 1999: 290), scrive sul Corriere della Sera nel dicembre 1973, poiché la propaganda consumistica ed edonistica dissimula il proprio autoritarismo rifacendosi a valori falsamente permissivi e ostentando una "tolleranza" che andrebbe invece considerata «la peggiore delle repressioni della storia umana» (Ibidem). Come ha notato Foucault ${ }^{8}$, il tratto distintivo del potere, e in particolare della sua declinazione odierna, è la "visibilità", da intendersi non come sinonimo di "trasparenza", bensì come subdola forma di sfruttamento massiccio degli spazi pubblici per insinuare il principio dell' autodisciplina: nelle società occidentali, il potere è più invisibile laddove è maggiormente visibile, perché è proprio nella costante visibilità che esso applica le proprie tecniche persuasive e manipolatorie.

Durante un'intervista rilasciata sul set di Salò, Pasolini commenta così l'affermazione del tirannico Duca sull'anarchia del potere:

Nulla è più anarchico del potere, il potere fa praticamente ciò che vuole. E ciò che il potere vuole è completamente arbitrario o dettato da sua necessità di carattere economico, che sfugge alle logiche razionali. Io detesto soprattutto il potere di oggi. Ognuno odia il potere che subisce, quindi odio con particolare veemenza il potere di questi giorni. È un potere che manipola i corpi in un modo orribile, che non ha niente da invidiare alla manipolazione fatta da Himmler o da Hitler. ${ }^{9}$

\footnotetext{
${ }^{8}$ Cfr. Foucault Michel (1994), Poteri e strategie. L'assoggettamento dei corpi e l'elemento sfuggente, Milano, Mimesis; FouCAult Michel (2014), La cura di sé. Storia della sessualità 3, Milano, Feltrinelli.

${ }^{9}$ L'intervista è riportata nel film di BerTOLUCCI Bernardo, Pasolini prossimo nostro (2006) e trascritta nel volume Bachmann Gideon, Pasolini Pier Paolo, Pier Paolo Pasolini. Polemica
} 
Le dichiarazioni del regista sono curiosamente in sintonia con le tesi di Foucault sul bio-potere, il quale, essendo «uno degli elementi indispensabili allo sviluppo del capitalismo» (FOUCAULT, 2013: 124), si è rivelato capace di una forgiatura inedita sulle personalità dei governati e di un «inserimento controllato dei corpi nell'apparato di produzione» $(\text { Ibidem })^{10}$. La vicenda del suo ultimo, controverso film si pone così come una sorta di orribile esperimento sull'effetto del Potere sui giovani, che al termine vengono "premiati" con la deportazione a Salò o puniti con torture, mutilazioni e sevizie inenarrabili. Nelle loro 120 giornate di orrori domestici, i libertini smentiscono ripetutamente la triade su cui si regge l'ideologia fascista, cioè Dio (invocarlo o alludere a concetti vagamente religiosi comporta la condanna a morte), Patria (che si riduce alla declamazione di una canzonetta fascista, mentre è in corso un atto di sodomia) e Famiglia (oltre al già citato matrimonio dei quattro fascisti con le reciproche figlie, il Duca pronuncia un elogio sperticato del matricidio). Il vincolo familiare che già in Pallavicino sbiadiva a fronte delle strategie del Re, il quale arriva a disporre del corpo della figlia stravolgendone l'autocoscienza, è qui completamente castrato in virtù di un sadismo impietoso. Le forme di ribellione timidamente abbozzate da alcuni prigionieri (che si traducono in pratiche sessuali non imposte dai gerarchi e in blande forme di solidarietà e d'affetto), vengono arginate quasi subito grazie a un subdolo incitamento alla delazione. L'esplorazione pasoliniana dell'oscenità del potere si traduce nella rappresentazione di un paradossale e brutale rovesciamento della morale pubblica dominante, imposto dagli stessi promulgatori di tale morale. Come sottolinea PASSANNANTI (2008: 31), «Il paradosso dell'osceno, in Salò, come delle 120 giornate sadiane, è che l'immoralità è imposta ai più deboli proprio da chi detiene il controllo della morale pubblica, minacciando in tal modo dall'interno gli stessi valori che sanziona». D'altro canto, nell'interpretazione pasoliniana, la religione non può coesistere con l'ideologia edonistica, «essendo quest'ultima il contrario della religione» (PASOLINI, 1999: 280), che abdica così al machiavelliano ruolo di instrumentum regni volto a «mascherare il proprio sostanziale illiberalismo e la propria sostanziale antidemocraticità affidando la funzione illiberale alla Chiesa, accettata in malafede come superiore istituzione religiosa» (Ibidem).

Inoltre, come nel Principe ermafrodito, sono presenti ripetute pratiche di crossdressing, ossia di travestimenti e scambi di ruoli di genere: durante la ributtante cena coprofaga, un giovane viene vestito da sposa e obbligato all'unione con uno dei gerarchi; i gerarchi stessi compaiono, nell'ultima sezione del film (il "Girone del sangue"), vestiti da nobildonne, in vista di una serie di matrimoni farseschi con alcuni soldati. In Salò, però, il clima vagamente comico e grottesco di Pallavicino è sostituito da un disgusto inquietante, che raggiunge l'acme nel "Girone della merda", un passaggio cruciale per comprendere il senso della

politica potere. Conversazione con Gideon Bachmann, ed. R. Costantini, Milano, Chiarelettere, p. 125-133.

${ }^{10}$ Per un approfondimento sulle concordanze tra Pasolini e Foucault in merito alla teorizzazione dello sfruttamento del corpo nella società consumistica cfr. D' Agostino Gianna (2016), Pensiero corsaro, Milano, Effigie. 
provocatoria operazione pasoliniana. L'oscenità del potere contemporaneo è, infatti, interamente riassumibile nella metafora fecale:

Salò, pertanto, allegorizza, con la "merda", la cecità del popolo alle manipolazioni messe a punto dal potere, a tutti i livelli dell'amministrazione della cosa pubblica, e, in maniera forse più diretta, esplicita, tematizza il consumismo quale aberrazione del comportamento delle masse, forma responsabile del regresso culturale che porta le società capitalistiche a monetizzare le proprie scorie, riammettendole nel circolo della produzione. (PASSANNANTI, 2008: 60)

Ed è soprattutto attraverso la repellente rappresentazione di questo pasto sui generis che Pasolini sfida l'industria culturale di cui pure ha avuto bisogno per realizzare il film, essendo uno dei momenti che mette maggiormente alla prova la capacità di resistenza dell'attonito fruitore dell'opera: «Pasolini conduce, pertanto, un doppio gioco, volto a porre il pubblico dinanzi all'impasse di un'opera che, nel darsi, si nega come valore morale ed estetico» (Ivi: 62); in altre parole, la sua sfida al potere si attua attraverso gli stessi strumenti di (dis)simulazione che connotano il potere.

Dopo l'azione ingannevole ed efferata del duca Valentino e la corte di frodi e menzogne di Pallavicino, nelle mani dei quattro Principi di Pasolini la crudeltà machiavelliana è sottoposta a una radicale perdita di senso, dato che essa non è più finalizzata alla costruzione del potere, ma diventa uno strumento sterile $\mathrm{e}$ autoreferenziale; diventa, cioè, un mezzo di godimento edonistico e di autocompiacimento narcisistico, volto ad appurare fin dove il potere "osceno", nascosto al pubblico, possa spingersi. Il giovane e audace Principe che nel penultimo capitolo dell'omonima opera cercava di vincere la Fortuna battendola con impeto, si è trasformato, nella società di massa, nel farsesco e meschino leviatano a quattro teste, che ha introiettato a tal punto la logica consumistica da arrivare a consumare, letteralmente, anche i corpi dei propri sudditi.

\section{BIBLIOGRAFIA}

ARNAUdO Marco (2009), L'altra dissimulazione: Accetto, Pallavicino, Machiavelli, Italica 86, p. 488-499.

ACCETTO Torquato (1997), Della dissimulazione onesta, ed. S. NIGRO, Torino, Einaudi.

Bachmann Gideon ; Pasolini Pier Paolo (2015), Pier Paolo Pasolini. Polemica politica potere. Conversazione con Gideon Bachmann, ed. R. CostANTINI, Milano, Chiarelettere.

BALDINI Enzo ; BATTISTA Annamaria (1997), Il dibattito politico nell'Italia della controriforma: Ragion di Stato, machiavellismo, utopia, Il pensiero politico 30, p. 393-439.

BATAILlE Georges (2006), La letteratura e il male, trad. it. A. ZANZOTTO, Milano, SE. 
Croce Benedetto (1928), Torquato Accetto e il trattatello Della dissimulazione onesta, in : CROCE Benedetto (1931), Nuovi saggi sulla letteratura italiana del Seicento, Bari, Laterza, p. 84-92.

D’Agostino Gianna (2016), Pensiero corsaro, Milano, Effigie.

DE SADE François (2014), Le 120 venti giornate di Sodoma, ed. M. CAVALLI, Milano, Rizzoli.

DonAldson Peter Samuel, Machiavelli and Mystery of State, Cambridge, Cambridge University Press.

FoucAult Michel (1994), Poteri e strategie. L'assoggettamento dei corpi e l'elemento sfuggente, Milano, Mimesis.

FouCAult Michel (2013), La volontà di sapere. Storia della sessualità 1, Milano, Feltrinelli.

Foucault Michel (2015), L’uso dei piaceri. Storia della sessualità 2, Milano, Feltrinelli.

FoucAult Michel (2014), La cura di sé. Storia della sessualità 3, Milano, Feltrinelli.

GENTILE Roberto (1995), Il potere senza volto. Un contributo psicologico allo studio dell'angoscia sociale, Milano, Franco Angeli.

GNOLI Antonio ; SASSO Gennaro (2013), I corrotti e gli inetti: conversazioni su Machiavelli, Milano, Bompiani.

INGLESE Giorgio (2006), Per Machiavelli: l'arte dello stato, la cognizione delle storie, Roma, Carocci.

LIPSIO Giusto (1604), Della politica ovvero dottrina civile libri VI dove si tratta del governo che devono fare i Principi per utilità dei sudditi e dell'obedienza e fedeltà che devono havere li Vassalli verso i loro Principi, si come anco del governare e mantenere guerre, trad. it., G. MARTINELLI, Roma, Guglielmo Martinelli, Giovanni Facciotti.

LODATO Saverio ; SCARPINATO Roberto (2008), Il ritorno del Principe, Milano, Chialettere.

MaChIAVElli Niccolò (1997), Opere. Vol. I: Gli scritti politici, ed. C. VIVANTI, Torino, Einaudi.

MACHIAVElli Niccolò (2013), Il Principe, a cura di G. INGLESE, Torino, Einaudi.

MARCHAND Jean-Jacques (1969), L'évolution de la figure de César Borgia dans la pensée de Machiavel, Revue Suisse d'Histoire 29, p. 327-355.

Murri Serafino (2007), Pier Paolo Pasolini. Salò o le 120 giornate di Sodoma, Torino, Lindau.

ORTOlani Donata (1978), Potere e violenza nel romanzo del Seicento, Catania, Pellicano.

PALlaVicino Ferrante (2005), Il principe ermafrodito, ed. R. Colombi, Roma, Salerno Editrice.

PASOlNi Pier Paolo (1999), Saggi sulla politica e sulla società, Milano, Mondadori.

PASOLINI Pier Paolo (2015), Salò o le 120 giornate di Sodoma, Bologna, Cineteca di Bologna.

PASSANNANTI Erminia (2008), Il corpo \& il potere. Salò o le centoventi giornate di Sodoma di Pier Paolo Pasolini, Novi Ligure (AL), Joker. 
QuONDAM Amedeo (2009), Introduzione, CASTIGLIONE Baldassar, Il Libro del Cortegiano, Milano, Garzanti.

SASSO Gennaro (1966), Machiavelli e Cesare Borgia. Storia di un giudizio, Roma, Edizioni dell'Ateneo.

SENNELART Michel (1989), Machiavélisme et raison d'État (XII ${ }^{\mathrm{e}}-\mathrm{XVIII}{ }^{\mathrm{e}}$ siècle), Paris, Presse Universitaire de France.

TABUCCHI Antonio (1998), La gastrite di Platone, Palermo, Sellerio.

VILlARI Rosario (1987), Elogio della dissimulazione. La lotta politica nel Seicento, Bari, Laterza.

Voza Pasquale (2016), Pasolini e la dittatura del presente, San Cesario di Lecce (LE), Manni Editori. 